\title{
Trends and correlates in meat consumption patterns in the US adult population
}

\author{
Youfa Wang ${ }^{1,2, *}$, May A Beydoun ${ }^{1,3}$, Benjamin Caballero', Tiffany L Gary ${ }^{2}$ \\ and Robert Lawrence ${ }^{4}$ \\ 'Department of International Health, Center for Human Nutrition, Johns Hopkins Bloomberg School of Public \\ Health, 615 N Wolfe Street, Baltimore, MD 21205, USA: ${ }^{2}$ Department of Epidemiology, Johns Hopkins \\ Bloomberg School of Public Health, Baltimore, MD, USA: ${ }^{3}$ National Institute on Aging, NIH/IRP, USA: \\ ${ }^{4}$ Department of Environmental Health Sciences, Center for a Livable Future, Johns Hopkins Bloomberg School of \\ Public Health, Baltimore, MD, USA
}

Submitted 25 February 2009: Accepted 13 January 2010: First published online 1 March 2010

\begin{abstract}
Objective: Few studies have examined recent shifts in meat consumption (MC), differences among US population groups, and the influence of psychosocialbehavioural factors.

Design: Nationally representative data collected for US adults aged $\geq 18$ years in the 1988-1994 and 1999-2004 National Health and Nutrition Examination Survey (NHANES) and the 1994-1996 Continuing Survey of Food Intakes by Individuals (CSFII) and Diet and Health Knowledge Survey (DHKS) were used.

Results: We found a U-shaped trend in MC, a decrease between 1988-1994 and 1994-1996, and an increase from 1994-1996 to 1999-2004. NHANES 1988-1994 and 1999-2004 indicate that MC did not change significantly, particularly for all meat, red meat, poultry and seafood. Between 1994-1996 and 1999-2004, average MC, including red meat, poultry, seafood and other meat products, increased in men. Women's total MC decreased, mainly due to decreased red meat and other meat products, except for increased seafood. Noticeable differences existed in the changes across population groups. Black men had the largest increase in consumption of total meat, poultry and seafood; Mexican American men had the smallest increase in poultry, seafood and other meat products. In 1999-2004, ethnic differences in MC became greater in women than among women in 1994-1996. Associations between MC and energy intake changed over time. Perceived benefit of dietary quality and food label use were associated with reduced red MC.

Conclusions: Noticeable differences exist in the shifts in MC across population groups and surveys. MC increased in men but decreased in women in recent years.
\end{abstract}

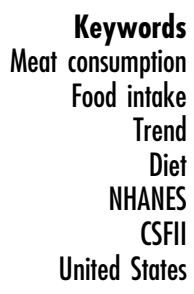

Meats are key dietary sources for many important nutrients including protein and many other essential micronutrients such as minerals and vitamins to maintain good health ${ }^{(1)}$. During recent years, the Atkins diet, which consists of high meat consumption (MC), has been advocated for weight loss ${ }^{(2,3)}$. On the other hand, MC has been linked to higher intakes of saturated fat, total energy and reduced consumption of vegetables ${ }^{(4,5)}$, leading some researchers to propose vegetarian diets as a means to prevent many diet-related chronic diseases including obesity, despite the many associated controversies ${ }^{(4,6-10)}$. MC has been related to increased chronic morbidity, while higher intake of vegetables, fruits, cereals, nuts and legumes has been independently associated with a lower risk for several chronic diseases, such as IHD, diabetes, obesity and many cancers ${ }^{(6,7)}$. Earlier studies indicate that diets largely based on plant foods, including well-balanced vegetarian diets, offer a number of nutritional benefits, including lower levels of saturated fat, cholesterol and animal protein, as well as higher levels of fibre, magnesium, potassium, folate and antioxidants. Compared to their non-vegetarian counterparts, vegetarians are found to have lower BMI, blood pressure and blood cholesterol levels; lower rates of hypertension, type 2 diabetes, prostate and colon cancer; and reduced death rates from heart disease ${ }^{(4,6,7)}$. Therefore, it would be of interest to examine MC patterns over time and determine whether they have been affected by the recent related research findings and advocacy campaigns, although many remain controversial ${ }^{(1,7,11,12)}$.

Using US nationally representative data collected over the past two decades, we examined the trends in US adults' MC and compared the differences among gender, ethnic, and socio-economic status (SES) groups. We also 
tested whether psychosocial-behavioural factors such as nutrition-related knowledge and perceptions, and food label use might affect MC. Our findings will help shed light on the recent shifts in food consumption patterns and the variation in these shifts across population groups in the United States.

\section{Materials and methods}

\section{Overview of study design}

Cross-sectional data collected from three data sets for adults (aged $\geq 18$ years) were used in our analysis: two rounds of the National Health and Nutrition Examination Survey (NHANES III 1988-1994 and 1999-2004), and the 1994-1996 Continuing Survey of Food Intakes by Individuals (CSFII). The dietary data ( $24 \mathrm{~h}$ recalls) collected in CSFII and NHANES were designed to provide national estimates of Americans' dietary intake patterns at the time of the survey. Thus, in theory their findings should be comparable, and could indicate time trends in Americans' dietary intakes. We chose to give more attention to the CSFII (1994-1996) than the NHANES III (1988-1994) considering that CSFII provided more recent data and two $24 \mathrm{~h}$ recalls while NHANES III had only one, and that CSFII collected information on participants' nutrition/ health knowledge and perception and other behavioural factors related to food consumption, whereas NHANES did not. In a $24 \mathrm{~h}$ dietary recall, the respondents were asked to report the kinds and amount of food and beverage items they ate over the past $24 \mathrm{~h}$. Subsequently, the data were linked to a food consumption table to help estimate nutrient and total energy intake.

\section{Database}

The NHANES include a series of cross-sectional surveys that provide nationally representative information on the nutrition and health status of the US civilian population. The National Center for Health Statistics (NCHS) conducted three rounds of NHANES surveys, in 1971-1975, 1976-1980 and 1988-1994, respectively. Since 1999, NHANES has been a continuous survey, and since 2002 the NHANES and CSFII dietary intake surveys were merged into a single inter-agency survey conducted jointly by NCHS and the US Department of Agriculture (USDA). Thus, the CSFII 1994-1996 and the NHANES 2003-2004 surveys used similar methodology. The data for the first 6 years of the period 1999-2004 were recently made available. Since 1999, the NHANES has included all people of all ages from birth, while some previous surveys excluded people aged 74 years or older. All NHANES used a stratified, multistage probability cluster sampling design, and collected data following standardised protocols. The survey consists of an in-home interview for demographic and basic health information and a health examination in a mobile examination centre. Household interviews were conducted by trained staff consisting of physicians, medical and health technicians, and dietary and health interviewers. Detailed descriptions of the study design and data collection have been published elsewhere $^{(13,14)}$.

The CSFII (1994-1996), conducted by the USDA, was designed to provide nationally representative information for assessing Americans' dietary intake patterns and trends over time ${ }^{(15)}$. A nationally representative multistage stratified sample of 16103 non-institutionalised persons aged 0-90 years residing in the United States provided information about dietary intake (by two $24 \mathrm{~h}$ recalls) in in-person interviews, but in some instances only one $24 \mathrm{~h}$ recall was obtained.

The Diet and Health Knowledge Survey (DHKS), a supplementary survey to CSFII, was completed by one adult per household selected ( $n$ 5765) among those aged $\geq 20$ years who had completed at least one $24 \mathrm{~h}$ recall in CSFII. DHKS included a number of questions that attempted to measure participants' knowledge, beliefs and perceptions related to nutrition and health, as well as their food habits and use of food labels in food purchase.

\section{Study populations}

The NHANES data collected in 1999-2000, 2001-2002 and 2003-2004 from US adults were merged and yielded a sample of 17061 participants (8091 men and 8970 women) that had complete demographic data. Only 15006 of them had complete dietary data (7148 men and 7858 women). NHANES III provided complete dietary data for 19618 adults; these were used in our analysis. In the CSFII 1994-1996, 10164 adults with two complete $24 \mathrm{~h}$ recalls ( 5198 men and 4966 women) were included.

To study the association between nutrition knowledge and psychosocial factors and MC using the CSFII/DHKS data, we excluded those aged 65 years or older ( $n$ 1319), to obtain a relatively healthy sample of individuals who were not on special diets, and excluded those who completed only one $24 \mathrm{~h}$ dietary recall $(n 90)$. This resulted in a final sample of 4356 individuals (2219 men and 2137 women) who completed both surveys (CSFII and DHKS).

\section{Dietary measures}

In CSFII and NHANES, $24 \mathrm{~h}$ dietary recalls were collected. In CSFII, dietary intake was assessed by one or two nonconsecutive, multiple-pass $24 \mathrm{~h}$ recalls that were $3-10 \mathrm{~d}$ apart. In earlier waves of NHANES (1988-1994 and 1999-2002), only one $24 \mathrm{~h}$ recall was collected from each participant in the household interview. A second day of recall was collected from all participants in NHANES 2003-2004. Energy and nutrient intakes were calculated by the USDA for CSFII and for NHANES 2003-2004, and by the Centers for Disease Control and Prevention/NCHS for NHANES 1999-2002. Major food groups including meats and vegetables and fruits (VF) were created by the USDA for the CSFII data ${ }^{(16)}$ (see below). We created these 
food groups for NHANES using similar methodology based on available food group codes for individual food items. These food groups (measured in grams) were used in our analysis. When two $24 \mathrm{~h}$ recalls were available, the averages were used. To our knowledge, the USDA system disaggregated mixed dishes into individual components for CSFII; however, NHANES grouped mixed dishes based on the main components rather than disaggregating them into individual food components. Thus, this might affect the comparability of the CSFII and NHANES food group data and our related findings.

\section{Meat groups}

In the CSFII data, the USDA grouped the different meats and meat products into the following groups: total meat (including all animal source food), red meat, poultry, seafood, and other meat products. For example, red meat consisted of beef, pork, lamb, veal and game. Poultry included chicken, turkey, duck and other poultry. Seafood included fish and shellfish. Other meat products included frankfurter and sausage, organ meats and food mixtures, mainly composed of meat, poultry and fish. Mixtures included items such as beef stew, spaghetti with meat sauce, chilli, sausage with gravy, soup with added meats, beef and potatoes or noodles, beef with rice and cooked vegetables, ham or shrimp or tuna salads, hamburgers or cheeseburgers, ham and cheese, roast beef or steak or chicken sandwiches and seafood mixtures. For simplicity, we call the combined meat groups 'all meat' in contrast to all plant source food.

\section{Nutrition-related psychosocial-bebavioural factors - food purchasing factors, nutrition knowledge, food label-related practices and food babits}

A number of questions included in the CSFII/DHKS asked about participants' knowledge, beliefs and perceptions related to nutrition and health. We chose six individual questions related to food purchasing behaviour measured on a 4-point Likert scale ('very important' to 'not important at all') enquiring about each participant's concern about 'how safe the food is to eat', 'its nutritional value', 'its price', 'how well the food keeps', 'how easy the food is to prepare' and 'its taste' and eight scales that covered areas of perceived benefit of diet quality ${ }^{(17)}$ (or how important it is for participants to follow general dietary guidelines: e.g. how important it is to purchase foods that are low in fat), food label-related practices (use, looking at, understanding and confidence in use), and food habits (e.g. choosing low-fat variety of foods or meat-related healthy habits, such as trimming fat from red meat and removing skin from poultry).

First, we conducted exploratory factor analysis to decide which scales in the DHKS could be reduced to a single factor that explained the majority of the variance in its manifest variables, using the Kaiser rule (i.e. eigenvalue $>1$ ). Next these scales were used to assess their associations with MC. Principal components analysis of variables within each scale was used for data reduction purposes. All extracted components (standardised Z-scores) were according to better health-related knowledge and food label practices and were named based on the content of each scale within the DHKS questionnaire. One component per scale was extracted and explained $40-70 \%$ of the variance in the measures included within each scale. We hypothesised that better practices and knowledge would be associated with less MC, particularly red meat. We also hypothesised that concern about price would reduce the consumption of food in general, including meat, and that concern about nutrition would have a similar effect on perceived benefit of diet quality.

\section{Main covariates}

\section{SES variables}

We used education and family income as the indicators of SES, considering their strengths and limitations ${ }^{(18)}$. Education was measured by years completed and then grouped into 0: '<High school education', 1: 'High school' (12 years) and 2: '> High school education'. The poverty income ratio (PIR) is the ratio of household income and the poverty line published by the Census Bureau for a certain family size in that calendar year. Specifically, we used the PIR categories of $0-100 \%$ (below the poverty line), $101-199 \%$ and $\geq 200 \%$.

\section{Other main food groups}

In our analysis, we used the consumption (in grams) of $\mathrm{VF}$ and grains as comparison for MC as well as potential confounders when assessing the association between MC and total energy intake. Fruits included whole fruit, dried and mixed dishes, and 100\% fruit juice; and vegetables included potatoes, fried potatoes, garden vegetables, salad greens and legumes.

\section{Other covariates}

Age, gender and race/ethnicity were considered as potentially confounding variables in our models. On the basis of the self-reported race and ethnicity, the participants were categorised as non-Hispanic (NH) whites, $\mathrm{NH}$ blacks, Mexican American (MA) and other.

\section{Statistical analysis}

First, we compared the average consumption of all meat and the meat groups over time, in the whole population and by gender. We also compared the changes across ethnic and SES groups. To test the differences by ethnicity and SES while controlling for other potential confounders, we fit linear regression models by including these variables simultaneously. Separate models were fit for each survey and for men and women. Next, using the CSFII/DHKS data and linear models, we tested the associations between MC and nutrition knowledge, psychosocial, food purchase and food habit factors. Finally, using linear regression analysis, we tested how meat, VF and grain consumption might be 
associated with total energy intake (TEI), the over-time changes in the associations and how much of the variance in TEI could be explained by them. All analyses were conducted using survey commands in the STATA statistical software package version 9.0 (Stata Corp., College Station, TX, USA) to account for the complex sample design effects in order to achieve nationally representative estimates and unbiased statistical inference ${ }^{(19)}$.

\section{Results}

\section{Trends in meat consumption and the differences across gender, ethnicity and socio-economic status}

These nationally representative data showed a U-shaped trend in MC between 1988-1994 and 1999-2004 (Table 1). The increase between 1994-1996 and 1999-2004 was due to increased MC by men for all the animal food groups. Women's MC decreased, which was driven by a decline in consumption of red meat and other meat products, while consumption of seafood increased. All these changes were statistically significant $(P<0 \cdot 05)$. Gender differences in average MC were significant only for 'all meat' in 1994-1996, but by 1999-2004 all became significant $(P<0 \cdot 05)$. However, when comparing the NHANES III (1988-1994) with 1999-2004 data, no significant consumption change was observed for 'all meat', red meat, poultry or seafood.

Table 2 compares MC trends across ethnic, gender and SES groups. A large proportion overall (62 of 200) of the time-dependent changes was significant $(P<0 \cdot 05)$, and most consisted of an increase among men and a decrease among women. Between 1994-1996 (CSFII) and 1999-2004 (NHANES), there were a number of noticeable differences in the changes across ethnic and SES groups. For example, NH-black men had the largest increase in the intakes of total meat, red meat, poultry and seafood. MA men had the smallest increase in the consumption of poultry, seafood and other meat products, though their increase in red MC was comparable to the two other major ethnic groups. Ethnic differences were more apparent among women: NHwhite women reduced consumption of all meat groups, particularly other meat products, while NH-black women increased consumption of all meat groups except for other meat products. The pattern of shift among MA women was not significant, with one exception wherein the reduction in other meat products was larger than that for their ethnic counterparts. Women with low education had the largest reduction during this period in consumption of other meat products, while women with high education reduced red MC. Interestingly, among women, only the high-income group reduced their red MC and increased their seafood consumption significantly.

Comparing NHANES III to 1999-2004 data, very few changes across race/ethnicity and SES groups were significant. A significant reduction in red MC was noted among NH-black men. MA women and men and women in the middle-income group had significant drops in consumption of other meat products. In general, differences in the changes among SES groups were small and inconsistent. Among men, most groups had increased their consumption of the various meat groups.

\section{Covariates of meat consumption}

Using linear regression models, we tested the cross-sectional associations between age, ethnicity and SES and total MC (Table 3), controlling for potential confounders. Our analysis indicates some noticeable gender and time (or survey) differences in the associations. Both waves of NHANES data show a consistent linear trend of decreased MC with age, while in CSFII, older groups consumed more meat than the reference age group. According to the NHANES men consumed more meat than women, by approximately $100 \mathrm{~g} / \mathrm{d}$, but only by $9 \mathrm{~g} / \mathrm{d}$ according to the CSFII.

Ethnic differences in MC changed over time. In general, they were disappearing in men (none remained significant in 1999-2004), but became stronger in women with NH-black women and MA women who consumed a much higher amount than white women in 1999-2004, by $46 \cdot 3 \mathrm{~g} / \mathrm{d}$ and $31 \cdot 4 \mathrm{~g} / \mathrm{d}$, respectively.

The association between SES and MC was weak. None of the tested associations were significant, except for that between education and MC among men in 1999-2004 men with a high school education had lower MC (by $26.6 \mathrm{~g} / \mathrm{d}$ ) than men with low education.

of the psychosocial-behavioural factors we tested while controlling for various demographic and SES factors, only a few were associated with total and subtypes of MC (Table 4). Only twelve of the eighty tests were significant. For example, those who weighed taste more as a factor in food selection consumed more red meat while those who used food labels consumed less.

\section{Association between meat consumption and total energy intake}

We also estimated the contribution of MC to TEI and the differences across food groups by adding the same amount $(100 \mathrm{~g} / \mathrm{d})$ of different food groups as well as the variation explained by these food groups and the changes between the survey periods (Table 5). A main finding was an inverted U-shaped relation between MC and TEI over time, both for the amount of energy contributed per $100 \mathrm{~g}$ of meat (i.e. overall energy density of meat) and for the variance in TEI explained by MC. In addition, the results showed that red meat contributed much more energy than other food groups, particularly VF. While VF accounted for $<6 \%$ of variation in TEI, MC explained $>10 \%$.

\section{Discussion}

Our findings based on nationally representative data indicate a U-shaped trend in US adults' MC between 
Table 1 Time trends in Americans' meat consumption (g/d/person) based on different national food surveys: 1988-1994 to 1999-2004

\begin{tabular}{|c|c|c|c|c|c|c|c|c|c|c|c|c|c|c|c|}
\hline & \multicolumn{2}{|c|}{$\begin{array}{l}\text { NHANES III } \\
\text { 1988-1994 }\end{array}$} & \multirow[b]{2}{*}{$P$ value } & \multicolumn{2}{|c|}{ CSFII 1994-96 } & \multirow[b]{2}{*}{$P$ value } & \multicolumn{2}{|c|}{$\begin{array}{l}\text { NHANES } \\
\text { 1999-2004 }\end{array}$} & \multirow[b]{2}{*}{$P$ value } & \multicolumn{6}{|c|}{ Changes } \\
\hline & Mean & SE & & Mean & SE & & Mean & SE & & $\begin{array}{c}1988-1994 \text { to } \\
1994-1996\end{array}$ & $P$ value & $\begin{array}{c}1994-1996 \text { to } \\
1999-2004\end{array}$ & $P$ value & $\begin{array}{c}1988-1994 \text { to } \\
1999-2004\end{array}$ & $P$ value \\
\hline Men and women & \multicolumn{2}{|c|}{$n 19618$} & & \multicolumn{2}{|c|}{$n 10164$} & & \multicolumn{2}{|c|}{$n 15006$} & & & & & & & \\
\hline All meat & $219 \cdot 0$ & $3 \cdot 8$ & & $181 \cdot 0$ & 1.9 & & $212 \cdot 4$ & $2 \cdot 5$ & & $-38 \cdot 0$ & * & $31 \cdot 4$ & * & $-6 \cdot 6$ & NS \\
\hline Red meat & $45 \cdot 4$ & $1 \cdot 3$ & & $32 \cdot 3$ & $0 \cdot 6$ & & 39.9 & $0 \cdot 8$ & & $-13 \cdot 1$ & * & $7 \cdot 6$ & * & $-5 \cdot 5$ & NS \\
\hline Poultry & $31 \cdot 7$ & $1 \cdot 4$ & & $22 \cdot 8$ & $0 \cdot 6$ & & $30 \cdot 0$ & $0 \cdot 8$ & & $-8 \cdot 9$ & * & $7 \cdot 2$ & * & $-1 \cdot 7$ & NS \\
\hline Seafood & $17 \cdot 1$ & $1 \cdot 3$ & & $9 \cdot 9$ & 0.5 & & $14 \cdot 2$ & 0.7 & & $-7 \cdot 2$ & * & $4 \cdot 3$ & * & $-2 \cdot 9$ & NS \\
\hline $\begin{array}{l}\text { Other meat } \\
\text { products }\end{array}$ & $124 \cdot 7$ & $3 \cdot 0$ & & $112 \cdot 3$ & $1 \cdot 8$ & & $109 \cdot 4$ & $2 \cdot 4$ & & $-12 \cdot 4$ & * & $-2 \cdot 9$ & NS & $-15 \cdot 3$ & $*$ \\
\hline Men & \multicolumn{2}{|c|}{$n 9193$} & & \multicolumn{2}{|c|}{$n 5198$} & & \multicolumn{2}{|c|}{$n 7148$} & & & & & & & \\
\hline All meat & $272 \cdot 0$ & $6 \cdot 8$ & ** & $185 \cdot 2$ & $2 \cdot 5$ & $\star *$ & $260 \cdot 0$ & 3.9 & ** & $-86 \cdot 8$ & * & $74 \cdot 8$ & * & $-12 \cdot 0$ & NS \\
\hline Red meat & $62 \cdot 0$ & $2 \cdot 0$ & ** & $32 \cdot 7$ & $0 \cdot 8$ & NS & $52 \cdot 9$ & $1 \cdot 2$ & ** & $-29 \cdot 3$ & * & $20 \cdot 2$ & * & $-9 \cdot 1$ & NS \\
\hline Poultry & $37 \cdot 0$ & 1.9 & ** & $23 \cdot 3$ & 0.9 & NS & $34 \cdot 8$ & $1 \cdot 2$ & $\star \star *$ & $-13 \cdot 7$ & * & $11 \cdot 5$ & * & $-2 \cdot 2$ & NS \\
\hline Seafood & $20 \cdot 2$ & $1 \cdot 8$ & ** & $10 \cdot 2$ & $0 \cdot 6$ & NS & $16 \cdot 5$ & $1 \cdot 0$ & ** & $-10 \cdot 0$ & * & $6 \cdot 3$ & * & $-3 \cdot 7$ & NS \\
\hline $\begin{array}{l}\text { Other meat } \\
\text { products }\end{array}$ & $152 \cdot 2$ & $5 \cdot 3$ & ** & $115 \cdot 1$ & $2 \cdot 2$ & NS & $134 \cdot 9$ & $3 \cdot 6$ & ** & $-37 \cdot 1$ & * & $19 \cdot 8$ & * & $-17 \cdot 3$ & NS \\
\hline Women & \multicolumn{2}{|c|}{ n 10425} & & \multicolumn{2}{|c|}{ n 4966} & & \multicolumn{2}{|c|}{$n 7858$} & & & & & & & \\
\hline All meat & $171 \cdot 1$ & $2 \cdot 7$ & & $177 \cdot 1$ & $2 \cdot 6$ & & $168 \cdot 5$ & $2 \cdot 2$ & & $6 \cdot 0$ & NS & $-8 \cdot 6$ & NS & $-2 \cdot 6$ & NS \\
\hline Red meat & $30 \cdot 4$ & 0.9 & & 31.9 & $0 \cdot 8$ & & $28 \cdot 0$ & 0.9 & & 1.5 & NS & -3.9 & * & $-2 \cdot 4$ & NS \\
\hline Poultry & $26 \cdot 4$ & $1 \cdot 2$ & & $22 \cdot 4$ & $0 \cdot 6$ & & $25 \cdot 6$ & 0.8 & & $-4 \cdot 0$ & * & $3 \cdot 2$ & NS & -0.8 & NS \\
\hline Seafood & $14 \cdot 4$ & $1 . \overline{5}$ & & 9.5 & $0 \cdot 6$ & & $12 \cdot 1$ & 0.7 & & -4.9 & * & $2 \cdot 6$ & * & $-2 \cdot 3$ & NS \\
\hline $\begin{array}{l}\text { Other meat } \\
\text { products }\end{array}$ & $99 \cdot 8$ & $2 \cdot 7$ & & $109 \cdot 8$ & $2 \cdot 6$ & & $85 \cdot 8$ & $2 \cdot 1$ & & $10 \cdot 0$ & * & $-24 \cdot 0$ & * & $-14 \cdot 0$ & $*$ \\
\hline
\end{tabular}

NHANES, National Health and Nutrition Examination Survey: CSFII, Continuing Survey of Food Intakes by Individuals.

${ }^{*}$ Changes between the periods CSFII 1994-1996 and NHANES 1999-2004 or between NHANES III and NHANES 1999-2004 were statistically significant, based on comparing $95 \%$ Cl: $P<0.05$. NS, the diferences in intakes were signilicant based on companing $95 \%$ Cl: $P<0.05$. 
Table 2 Time trends in American men's and women's meat consumption (g/d/person) by types of meat, ethnicity and SES (education and income): 1988-1994 to 1999-2004

\begin{tabular}{|c|c|c|c|c|c|c|c|c|c|c|c|c|c|c|c|c|c|c|c|c|}
\hline & \multicolumn{8}{|c|}{ Ethnicity } & \multicolumn{6}{|c|}{ Education } & \multicolumn{6}{|c|}{ Poverty income ratio } \\
\hline & \multicolumn{2}{|c|}{$\mathrm{NH}$ white } & \multicolumn{2}{|c|}{$\mathrm{NH}$ black } & \multicolumn{2}{|c|}{ MA } & \multicolumn{2}{|c|}{ Other } & \multicolumn{2}{|c|}{$<\mathrm{HS}$} & \multicolumn{2}{|c|}{ HS } & \multicolumn{2}{|c|}{$>\mathrm{HS}$} & \multicolumn{2}{|c|}{ Low } & \multicolumn{2}{|c|}{ Medium } & \multicolumn{2}{|c|}{ High } \\
\hline & Mean & SE & Mean & SE & Mean & SE & Mean & SE & Mean & SE & Mean & SE & Mean & SE & Mean & SE & Mean & SE & Mean & SE \\
\hline \multicolumn{21}{|l|}{ Men } \\
\hline \multicolumn{21}{|l|}{ All meat } \\
\hline 1988-1994 & $263 \cdot 7$ & $8 \cdot 7$ & $309 \cdot 3$ & $6 \cdot 0$ & $270 \cdot 9$ & $6 \cdot 9$ & $305 \cdot 2$ & $20 \cdot 0$ & $261 \cdot 6$ & $12 \cdot 1$ & $281 \cdot 2$ & $11 \cdot 4$ & $265 \cdot 5$ & $7 \cdot 0$ & $297 \cdot 7$ & $17 \cdot 7$ & $279 \cdot 6$ & $13 \cdot 6$ & $267 \cdot 5$ & $7 \cdot 8$ \\
\hline 1994-1996 & $185 \cdot 3$ & $3 \cdot 0$ & $181 \cdot 7$ & $8 \cdot 3$ & $181 \cdot 0$ & $9 \cdot 5$ & $190 \cdot 6$ & $9 \cdot 2$ & $194 \cdot 3$ & $8 \cdot 7$ & $188 \cdot 4$ & $4 \cdot 3$ & $181 \cdot 2$ & $4 \cdot 0$ & $195 \cdot 8$ & $6 \cdot 7$ & $183 \cdot 7$ & $6 \cdot 5$ & $184 \cdot 2$ & $2 \cdot 7$ \\
\hline 1999-2004 & 254.9 & $4 \cdot 5$ & $281 \cdot 1$ & $8 \cdot 0$ & $275 \cdot 1$ & $8 \cdot 0$ & $264 \cdot 8$ & 13.9 & $270 \cdot 4$ & $7 \cdot 2$ & $268 \cdot 3$ & $6 \cdot 6$ & $251 \cdot 6$ & $5 \cdot 1$ & $258 \cdot 8$ & $10 \cdot 8$ & $261 \cdot 0$ & 8.5 & $260 \cdot 0$ & 3.9 \\
\hline$\Delta 1$ & 69 . & & 99 & & 94. & & 74 & & 76 . & & 79 & & $70 \cdot 2$ & & 63 & & $72 \cdot 3$ & & $75 \cdot 8$ & \\
\hline$\Delta 2$ & $-8 \cdot \varepsilon$ & & -28 & & $4 \cdot$ & & -40 & & 8 . & & -12 & & $-13 \cdot 5$ & & -38 & & $-18 \cdot 6$ & & $-7 \cdot 5$ & \\
\hline Red meat & & & & & & & & & & & & & & & & & & & & \\
\hline 1988-1994 & $58 \cdot 7$ & $2 \cdot 3$ & $63 \cdot 9$ & $3 \cdot 0$ & $69 \cdot 9$ & $4 \cdot 9$ & $86 \cdot 6$ & $9 \cdot 3$ & $59 \cdot 0$ & $4 \cdot 4$ & $69 \cdot 4$ & $3 \cdot 1$ & $54 \cdot 7$ & $2 \cdot 7$ & $69 \cdot 1$ & $4 \cdot 3$ & $60 \cdot 4$ & $4 \cdot 1$ & $61 \cdot 3$ & $2 \cdot 4$ \\
\hline 1994-1996 & $32 \cdot 7$ & 0.9 & $28 \cdot 1$ & $3 \cdot 0$ & $37 \cdot 7$ & $4 \cdot 5$ & $39 \cdot 3$ & $3 \cdot 5$ & $35 \cdot 1$ & $3 \cdot 3$ & $31 \cdot 6$ & $1 \cdot 1$ & $33 \cdot 3$ & $1 \cdot 3$ & $30 \cdot 7$ & $3 \cdot 2$ & $35 \cdot 4$ & $1 \cdot 9$ & $32 \cdot 3$ & 0.9 \\
\hline 1999-2004 & $52 \cdot 6$ & $1 \cdot 7$ & $51 \cdot 1$ & $2 \cdot 7$ & $60 \cdot 3$ & $4 \cdot 0$ & $50 \cdot 2$ & $4 \cdot 2$ & $54 \cdot 7$ & $2 \cdot 5$ & $57 \cdot 9$ & $2 \cdot 9$ & $49 \cdot 7$ & $1 \cdot 7$ & $52 \cdot 3$ & 3.4 & $49 \cdot 1$ & $3 \cdot 7$ & $53 \cdot 9$ & $1 \cdot 8$ \\
\hline$\Delta 1$ & 19 & & 23. & & $22 \cdot$ & & 10 & & 19. & & 26 & & $16 \cdot$ & & 21 . & & $13 \cdot 7$ & & $21 \cdot 6$ & \\
\hline$\Delta 2$ & -6 & & -12 & & $-9 \cdot$ & & -36 & & -4 . & & $-11 \cdot$ & & $-5 \cdot($ & & -16 & & -11.3 & & $-7 \cdot 4$ & \\
\hline Poultry & & & & & & & & & & & & & & & & & & & & \\
\hline 1988-1994 & $34 \cdot 4$ & $2 \cdot 2$ & $64 \cdot 7$ & $2 \cdot 7$ & $31 \cdot 4$ & $2 \cdot 3$ & $36 \cdot 7$ & $5 \cdot 2$ & $30 \cdot 1$ & $3 \cdot 6$ & $35 \cdot 1$ & $1 \cdot 8$ & $42 \cdot 2$ & $3 \cdot 4$ & $43 \cdot 1$ & $4 \cdot 8$ & $33 \cdot 2$ & $2 \cdot 2$ & $39 \cdot 0$ & $2 \cdot 7$ \\
\hline 1994-1996 & $23 \cdot 4$ & 0.9 & $21 \cdot 8$ & $2 \cdot 6$ & $25 \cdot 5$ & $3 \cdot 2$ & $23 \cdot 7$ & $4 \cdot 9$ & $23 \cdot 8$ & $2 \cdot 3$ & $21 \cdot 3$ & $1 \cdot 1$ & $24 \cdot 8$ & $1 \cdot 3$ & $23 \cdot 7$ & $2 \cdot 0$ & $22 \cdot 2$ & $2 \cdot 1$ & $23 \cdot 5$ & $1 \cdot 1$ \\
\hline 1999-2004 & $31 \cdot 4$ & $1 \cdot 3$ & $60 \cdot 8$ & 3.9 & $31 \cdot 1$ & $2 \cdot 4$ & $36 \cdot 1$ & $3 \cdot 8$ & $31 \cdot 9$ & $1 \cdot 7$ & $36 \cdot 7$ & $2 \cdot 2$ & $35 \cdot 0$ & $1 \cdot 8$ & $33 \cdot 3$ & $3 \cdot 1$ & $36 \cdot 9$ & $3 \cdot 0$ & $34 \cdot 5$ & $1 \cdot 2$ \\
\hline$\Delta 1$ & $8 \cdot 0$ & & $39 \cdot$ & & $5 \cdot 6$ & & 12 & & $8 \cdot 1$ & & 15 & & 10. & & $9 \cdot 6$ & & $14 \cdot 7$ & & $11 \cdot 0$ & \\
\hline$\Delta 2$ & $-3 \cdot 0$ & & -3 & & -0.3 & & -0 . & & $1 \cdot \varepsilon$ & & $1 \cdot($ & & $-7 \cdot 5$ & & $-9 \cdot \varepsilon$ & & $3 \cdot 7$ & & $-4 \cdot 5$ & \\
\hline Seafood & & & & & & & & & & & & & & & & & & & & \\
\hline 1988-1994 & $18 \cdot 4$ & $2 \cdot 1$ & $29 \cdot 1$ & $2 \cdot 3$ & $14 \cdot 1$ & $1 \cdot 8$ & $30 \cdot 7$ & $5 \cdot 1$ & $15 \cdot 9$ & $2 \cdot 8$ & $19 \cdot 6$ & $2 \cdot 5$ & $22 \cdot 1$ & $2 \cdot 6$ & $18 \cdot 7$ & $3 \cdot 1$ & $16 \cdot 8$ & $3 \cdot 7$ & $20 \cdot 5$ & $2 \cdot 2$ \\
\hline 1994-1996 & $10 \cdot 7$ & $0 \cdot 7$ & $6 \cdot 4$ & $1 \cdot 1$ & $11 \cdot 6$ & $4 \cdot 4$ & $9 \cdot 7$ & $2 \cdot 3$ & $9 \cdot 4$ & $2 \cdot 0$ & $11 \cdot 7$ & $1 \cdot 2$ & $9 \cdot 1$ & $0 \cdot 7$ & $10 \cdot 9$ & $1 \cdot 9$ & $8 \cdot 7$ & 0.9 & $10 \cdot 5$ & 0.8 \\
\hline 1999-2004 & $15 \cdot 0$ & $1 \cdot 3$ & $28 \cdot 2$ & $2 \cdot 8$ & $11 \cdot 6$ & $1 \cdot 2$ & $19 \cdot 9$ & $3 \cdot 0$ & $18 \cdot 6$ & $1 \cdot 8$ & $13 \cdot 6$ & $1 \cdot 5$ & $17 \cdot 2$ & $1 \cdot 2$ & $13 \cdot 1$ & $1 \cdot 8$ & $17 \cdot 4$ & $2 \cdot 4$ & $16 \cdot 9$ & $1 \cdot 1$ \\
\hline$\Delta 1$ & $4 \cdot 3$ & & $21 \cdot$ & & $0 . \mathrm{c}$ & & 10 & & $9 \cdot 2$ & & $1 \cdot 5$ & & $8 \cdot 1$ & & 2 & & $8 \cdot 7$ & & 6.5 & \\
\hline$\Delta 2$ & $-3 \cdot 4$ & & -0 & & $-2 \cdot 5$ & & $-10 \cdot \varepsilon$ & & $2 \cdot 7$ & & $-6 \cdot($ & & $-4 \cdot 9$ & & -5 & & $0 \cdot 6$ & & $-3 \cdot 6$ & \\
\hline Other meat pr & & & & & & & & & & & & & & & & & & & & \\
\hline 1988-1994 & $152 \cdot 2$ & $7 \cdot 0$ & $151 \cdot 6$ & $5 \cdot 0$ & $155 \cdot 3$ & $6 \cdot 3$ & $150 \cdot 9$ & $18 \cdot 1$ & $156 \cdot 5$ & $12 \cdot 0$ & $157 \cdot 0$ & $8 \cdot 8$ & $146 \cdot 4$ & $5 \cdot 6$ & $166 \cdot 7$ & $17 \cdot 2$ & $169 \cdot 0$ & $11 \cdot 1$ & $146 \cdot 6$ & $6 \cdot 5$ \\
\hline 1994-1996 & $114 \cdot 4$ & $2 \cdot 5$ & $123 \cdot 1$ & $10 \cdot 8$ & $103 \cdot 6$ & $7 \cdot 5$ & $113 \cdot 7$ & $6 \cdot 1$ & $123 \cdot 2$ & $8 \cdot 0$ & $120 \cdot 0$ & $3 \cdot 9$ & $110 \cdot 0$ & $3 \cdot 4$ & $127 \cdot 5$ & $10 \cdot 0$ & $112 \cdot 9$ & $5 \cdot 6$ & $114 \cdot 1$ & $2 \cdot 4$ \\
\hline 1999-2004 & $138 \cdot 8$ & $4 \cdot 2$ & $130 \cdot 8$ & $6 \cdot 4$ & $106 \cdot 1$ & $5 \cdot 4$ & $133 \cdot 6$ & $12 \cdot 4$ & $131 \cdot 4$ & $5 \cdot 8$ & $140 \cdot 8$ & 6.5 & $133 \cdot 0$ & $4 \cdot 9$ & $131 \cdot 8$ & $9 \cdot 3$ & $129 \cdot 3$ & $7 \cdot 3$ & $136 \cdot 9$ & $3 \cdot 6$ \\
\hline$\Delta 1$ & 24 & & $7 \cdot 7$ & & $2 \cdot 5$ & & 19 & & 8 & & 20 & & $23 \cdot$ & & -0 & & 16 & & $22 \cdot 8$ & \\
\hline$\Delta 2$ & -13 & & $-20 \cdot \varepsilon$ & & $-49 \cdot 2$ & & -17 & & -25 & & -16 & & -13 & & -34 & & -39 & & $9 \cdot 7$ & \\
\hline Women & & & & & & & & & & & & & & & & & & & & \\
\hline All meat & & & & & & & & & & & & & & & & & & & & \\
\hline 1988-1994 & $163 \cdot 2$ & $3 \cdot 0$ & $199 \cdot 8$ & $5 \cdot 2$ & $174 \cdot 8$ & $5 \cdot 1$ & $203 \cdot 5$ & $15 \cdot 9$ & $166 \cdot 0$ & $7 \cdot 8$ & $178 \cdot 0$ & $4 \cdot 3$ & $163 \cdot 3$ & $4 \cdot 2$ & $172 \cdot 1$ & $7 \cdot 4$ & $175 \cdot 5$ & $6 \cdot 4$ & $171 \cdot 2$ & 3.9 \\
\hline 1994-1996 & $175 \cdot 7$ & $3 \cdot 1$ & $193 \cdot 4$ & $9 \cdot 5$ & $189 \cdot 0$ & $17 \cdot 6$ & $155 \cdot 7$ & $8 \cdot 4$ & $174 \cdot 2$ & $7 \cdot 5$ & $176 \cdot 8$ & $3 \cdot 2$ & $177 \cdot 8$ & $4 \cdot 9$ & $171 \cdot 6$ & $5 \cdot 5$ & $176 \cdot 9$ & $5 \cdot 3$ & $178 \cdot 2$ & $3 \cdot 4$ \\
\hline 1999-2004 & $159 \cdot 4$ & $2 \cdot 7$ & $207 \cdot 6$ & $4 \cdot 7$ & $195 \cdot 0$ & $5 \cdot 7$ & $169 \cdot 4$ & $8 \cdot 8$ & $169 \cdot 7$ & $5 \cdot 1$ & $168 \cdot 9$ & $4 \cdot 5$ & $167 \cdot 8$ & $3 \cdot 6$ & $176 \cdot 4$ & $5 \cdot 8$ & $162 \cdot 3$ & $4 \cdot 1$ & $168 \cdot 5$ & $2 \cdot 9$ \\
\hline$\Delta 1$ & -16 & & 14 & & $6 \cdot c$ & & 13 & & -4 & & -7 & & -10 & & 4 & & -14 & & -9 & \\
\hline$\Delta 2$ & -3 & & $7 \cdot 8$ & & 20. & & -34 & & & $\cdot 7$ & -9 & & 4 & 5 & $4 \cdot$ & & -13 & & -2 & \\
\hline Red meat & & & & & & & & & & & & & & & & & & & & \\
\hline 1988-1994 & $29 \cdot 0$ & $1 \cdot 0$ & $35 \cdot 6$ & $1 \cdot 3$ & $36 \cdot 7$ & $2 \cdot 1$ & $32 \cdot 9$ & $3 \cdot 6$ & $30 \cdot 5$ & $2 \cdot 0$ & $32 \cdot 3$ & $1 \cdot 2$ & $27 \cdot 6$ & $1 \cdot 7$ & $34 \cdot 5$ & $2 \cdot 4$ & $32 \cdot 6$ & $1 \cdot 8$ & $28 \cdot 5$ & $1 \cdot 2$ \\
\hline 1994-1996 & $32 \cdot 5$ & 0.9 & $30 \cdot 0$ & $2 \cdot 1$ & $25 \cdot 7$ & $3 \cdot 0$ & $30 \cdot 3$ & $3 \cdot 0$ & $30 \cdot 4$ & $2 \cdot 1$ & $30 \cdot 1$ & $1 \cdot 1$ & $33 \cdot 9$ & $1 \cdot 5$ & $29 \cdot 7$ & $1 \cdot 9$ & $29 \cdot 3$ & $1 \cdot 3$ & $33 \cdot 2$ & $1 \cdot 1$ \\
\hline 1999-2004 & $26 \cdot 9$ & $1 \cdot 1$ & $34 \cdot 3$ & $2 \cdot 2$ & $35 \cdot 1$ & $1 \cdot 7$ & $24 \cdot 0$ & $2 \cdot 1$ & $30 \cdot 5$ & $1 \cdot 9$ & $30 \cdot 8$ & $1 \cdot 7$ & $25 \cdot 5$ & $1 \cdot 1$ & $27 \cdot 3$ & $1 \cdot 8$ & $27 \cdot 5$ & $1 \cdot 5$ & $28 \cdot 3$ & $1 \cdot 0$ \\
\hline$\Delta 1$ & -5 & & 4 & & 9 & & -6 & & 0 & & 0 & 7 & -8 & & -2 & & -1 & & -4 & \\
\hline$\Delta 2$ & -2 & & -1 & & -1 & & -8 & & 0 . & & -1 & & -2 & & -7 & & -5 & & -0 & \\
\hline
\end{tabular}


Table 2 Continued

\begin{tabular}{|c|c|c|c|c|c|c|c|c|c|c|c|c|c|c|c|c|c|c|c|c|}
\hline & \multicolumn{8}{|c|}{ Ethnicity } & \multicolumn{6}{|c|}{ Education } & \multicolumn{6}{|c|}{ Poverty income ratio } \\
\hline & \multicolumn{2}{|c|}{$\mathrm{NH}$ white } & \multicolumn{2}{|c|}{$\mathrm{NH}$ black } & \multicolumn{2}{|c|}{ MA } & \multicolumn{2}{|c|}{ Other } & \multicolumn{2}{|c|}{$<\mathrm{HS}$} & \multicolumn{2}{|c|}{ HS } & \multicolumn{2}{|c|}{$>\mathrm{HS}$} & \multicolumn{2}{|c|}{ Low } & \multicolumn{2}{|c|}{ Medium } & \multicolumn{2}{|c|}{ High } \\
\hline & Mean & SE & Mean & SE & Mean & SE & Mean & SE & Mean & $\mathrm{SE}$ & Mean & SE & Mean & SE & Mean & SE & Mean & SE & Mean & SE \\
\hline \multicolumn{21}{|l|}{ Poultry } \\
\hline 1988-1994 & $23 \cdot 4$ & $1 \cdot 4$ & $43 \cdot 4$ & $1 \cdot 9$ & $24 \cdot 0$ & 1.5 & $31 \cdot 6$ & $3 \cdot 1$ & $25 \cdot 8$ & $1 \cdot 8$ & $26 \cdot 0$ & $1 \cdot 3$ & $27 \cdot 3$ & $2 \cdot 0$ & $27 \cdot 8$ & $1 \cdot 9$ & $24 \cdot 4$ & $1 \cdot 7$ & $26 \cdot 9$ & $1 \cdot 9$ \\
\hline 1994-1996 & $22 \cdot 9$ & 0.6 & $20 \cdot 8$ & $2 \cdot 2$ & $20 \cdot 1$ & $4 \cdot 0$ & $20 \cdot 9$ & $2 \cdot 9$ & $22 \cdot 8$ & $1 \cdot 7$ & $21 \cdot 6$ & 0.9 & $23 \cdot 2$ & $0 \cdot 9$ & $19 \cdot 6$ & $1 \cdot 5$ & $20 \cdot 4$ & $1 \cdot 6$ & $23 \cdot 7$ & $0 \cdot 8$ \\
\hline 1999-2004 & $22 \cdot 2$ & 0.9 & $44 \cdot 3$ & $2 \cdot 0$ & $24 \cdot 2$ & $1 \cdot 2$ & $29 \cdot 2$ & $2 \cdot 3$ & $24 \cdot 9$ & $1 \cdot 4$ & $24 \cdot 0$ & $1 \cdot 6$ & $26 \cdot 8$ & $1 \cdot 0$ & $27 \cdot 7$ & $2 \cdot 5$ & $24 \cdot 3$ & $1 \cdot 9$ & $25 \cdot 6$ & $0 \cdot 7$ \\
\hline$\Delta 1$ & \multirow{2}{*}{\multicolumn{2}{|c|}{$\begin{array}{l}-0.7 \\
-1.2\end{array}$}} & \multirow{2}{*}{\multicolumn{2}{|c|}{$\begin{array}{c}23 \cdot 5^{\star} \\
0.9\end{array}$}} & \multirow{2}{*}{\multicolumn{2}{|c|}{$\begin{array}{l}4 \cdot 1 \\
0.2\end{array}$}} & \multirow{2}{*}{\multicolumn{2}{|c|}{$\begin{array}{r}8 \cdot 3 \\
-2 \cdot 4\end{array}$}} & \multirow{2}{*}{\multicolumn{2}{|c|}{$\begin{array}{r}2 \cdot 1 \\
-0.9\end{array}$}} & \multirow{2}{*}{\multicolumn{2}{|c|}{$\begin{array}{r}2.4 \\
-2.0\end{array}$}} & \multirow{2}{*}{\multicolumn{2}{|c|}{$\begin{array}{r}3.6 \\
-0.5\end{array}$}} & \multirow{2}{*}{\multicolumn{2}{|c|}{$\begin{array}{r}8 \cdot 1 \\
-0 \cdot 1\end{array}$}} & \multirow{2}{*}{\multicolumn{2}{|c|}{$\begin{array}{r}3.9 \\
-0.1\end{array}$}} & \multirow{2}{*}{\multicolumn{2}{|c|}{$\begin{array}{r}1.9 \\
-1.3\end{array}$}} \\
\hline$\Delta 2$ & & & & & & & & & & & & & & & & & & & & \\
\hline \multicolumn{21}{|l|}{ Seafood } \\
\hline 1988-1994 & $12 \cdot 3$ & $1 \cdot 6$ & $21 \cdot 6$ & $1 \cdot 6$ & $9 \cdot 6$ & $1 \cdot 4$ & $27 \cdot 2$ & $6 \cdot 5$ & $15 \cdot 1$ & $2 \cdot 8$ & $14 \cdot 3$ & $2 \cdot 0$ & $14 \cdot 3$ & $2 \cdot 1$ & $13 \cdot 8$ & $1 \cdot 9$ & $13 \cdot 2$ & $3 \cdot 1$ & $14 \cdot 3$ & $1 \cdot 6$ \\
\hline 1994-1996 & $9 \cdot 1$ & 0.7 & $12 \cdot 2$ & $2 \cdot 8$ & $9 \cdot 9$ & $3 \cdot 8$ & $9 \cdot 2$ & $1 \cdot 7$ & $8 \cdot 3$ & $1 \cdot 6$ & $10 \cdot 1$ & 0.9 & $9 \cdot 1$ & $0 \cdot 8$ & $7 \cdot 1$ & $0 \cdot 8$ & $10 \cdot 3$ & $1 \cdot 7$ & $9 \cdot 7$ & $0 \cdot 7$ \\
\hline 1999-2004 & $10 \cdot 2$ & 0.9 & $22 \cdot 5$ & $2 \cdot 3$ & $8 \cdot 5$ & $1 \cdot 0$ & $15 \cdot 8$ & $2 \cdot 3$ & $13 \cdot 2$ & $1 \cdot 2$ & $9 \cdot 9$ & $1 \cdot 1$ & $12 \cdot 8$ & $1 \cdot 0$ & $9 \cdot 6$ & $1 \cdot 4$ & $10 \cdot 9$ & $1 \cdot 3$ & $13 \cdot 1$ & $1 \cdot 0$ \\
\hline$\Delta 1$ & \multicolumn{2}{|c|}{$1 \cdot 1$} & \multirow{2}{*}{\multicolumn{2}{|c|}{$\begin{array}{c}10.3^{\star} \\
0.9\end{array}$}} & \multirow{2}{*}{\multicolumn{2}{|c|}{$\begin{array}{l}-1 \cdot 4 \\
-1 \cdot 1\end{array}$}} & & & $4 \cdot$ & & $-c$ & & $3 \cdot 7$ & & 2 & & 0 & & & \\
\hline$\Delta 2$ & -2 & & & & & & -1 & & -1 & & & & -1.5 & & -4 & & -2 & & -1 & \\
\hline Other meat pr & & & & & & & & & & & & & & & & & & & & \\
\hline 1988-1994 & $98 \cdot 4$ & $3 \cdot 4$ & $99 \cdot 0$ & $3 \cdot 9$ & $104 \cdot 5$ & $5 \cdot 9$ & $111 \cdot 7$ & $13 \cdot 7$ & $94 \cdot 5$ & $7 \cdot 3$ & $105 \cdot 3$ & $3 \cdot 9$ & $94 \cdot 0$ & $4 \cdot 0$ & $95 \cdot 8$ & $6 \cdot 6$ & $105 \cdot 0$ & $5 \cdot 4$ & $101 \cdot 4$ & $3 \cdot 9$ \\
\hline 1994-1996 & $107 \cdot 9$ & $3 \cdot 0$ & $126 \cdot 2$ & $7 \cdot 6$ & $131 \cdot 6$ & $20 \cdot 2$ & $92 \cdot 7$ & $7 \cdot 6$ & $108 \cdot 5$ & $7 \cdot 7$ & 111.9 & $3 \cdot 0$ & $108 \cdot 0$ & $4 \cdot 4$ & $112 \cdot 2$ & $4 \cdot 6$ & $114 \cdot 0$ & $4 \cdot 4$ & $108 \cdot 0$ & $3 \cdot 6$ \\
\hline 1999-2004 & $87 \cdot 0$ & $2 \cdot 6$ & $94 \cdot 8$ & $3 \cdot 8$ & $74 \cdot 1$ & $4 \cdot 1$ & $74 \cdot 8$ & $6 \cdot 4$ & $76 \cdot 9$ & $4 \cdot 0$ & $92 \cdot 3$ & $3 \cdot 7$ & $86 \cdot 3$ & 3.5 & $87 \cdot 8$ & $4 \cdot 5$ & $82 \cdot 7$ & 3.9 & $86 \cdot 4$ & $2 \cdot 6$ \\
\hline$\Delta 1$ & -20 & & -3 & & -5 & & -1 & & -31 & & -1 & & -21 & & -2 & & -31 & & -21 & \\
\hline$\Delta 2$ & -11 & & -4 & & -3 & & -3 & & -17 & & -1 & & -7 & & -8 & & -22 & & -15 & \\
\hline
\end{tabular}

SES, socio-economic status; NH, non-Hispanic; MA, Mexican American; HS, high school; $\mathbf{\Delta 1}$, change between 1994-1996 and 1999-2004; $\Delta 2$, change between 1988-1994 and 1999-2004.

${ }^{*}$ Changes between two periods (1994-1996 v. 1999-2004 or 1988-1994 v. 1999-2004) were statistically significant, based on comparing $95 \%$ Cl: $P<0 \cdot 05$. 
Table 3 Differences in total meat consumption by sex, age, ethnicity and SES (income and education): 1988-1994 to 1999-2004t, $\neq$

\begin{tabular}{|c|c|c|c|c|c|c|c|c|c|}
\hline & \multicolumn{3}{|c|}{ NHANES III 1988-1994 } & \multicolumn{3}{|c|}{ CSFII 1994-1996 } & \multicolumn{3}{|c|}{ NHANES 1999-2004 } \\
\hline & $\beta$ & SE & $P$ value & $\beta$ & SE & $P$ value & $\beta$ & SE & $P$ value \\
\hline \multicolumn{10}{|l|}{ Men and women } \\
\hline \multirow{2}{*}{\multicolumn{10}{|c|}{ Age group (years) }} \\
\hline & & & & & & & & & \\
\hline $30-49$ & -0.4 & $7 \cdot 2$ & 0.959 & $11 \cdot 8$ & $5 \cdot 2$ & $0.027^{\star *}$ & 0.9 & $5 \cdot 6$ & $0 \cdot 867$ \\
\hline $50-69$ & $-18 \cdot 5$ & $8 \cdot 4$ & $0.033^{\star \star}$ & $11 \cdot 6$ & $5 \cdot 5$ & $0.042^{\star \star}$ & $-24 \cdot 7$ & $6 \cdot 1$ & $0 \cdot 001^{* *}$ \\
\hline$>70$ & $-56 \cdot 5$ & $10 \cdot 1$ & $<0.001^{\star *}$ & $15 \cdot 3$ & $7 \cdot 3$ & $0.042^{\star *}$ & $-59 \cdot 5$ & $6 \cdot 0$ & $<0.001^{\star *}$ \\
\hline Female ( $v$. male) & $-100 \cdot 7$ & $7 \cdot 4$ & $<0.001^{\star *}$ & $-8 \cdot 7$ & $3 \cdot 4$ & $0 \cdot 016^{\star \star}$ & $-89 \cdot 4$ & $3 \cdot 9$ & $<0.001^{\star *}$ \\
\hline \multicolumn{10}{|l|}{ Ethnicity } \\
\hline \multicolumn{10}{|l|}{ NH white (Ref.) } \\
\hline $\mathrm{NH}$ black & $33 \cdot 6$ & $6 \cdot 8$ & $<0.001^{\star *}$ & $8 \cdot 3$ & $6 \cdot 2$ & $0 \cdot 187$ & $33 \cdot 0$ & $6 \cdot 1$ & $<0.001^{\star *}$ \\
\hline MA & $-2 \cdot 0$ & $8 \cdot 9$ & 0.824 & $7 \cdot 6$ & $12 \cdot 3$ & 0.540 & $16 \cdot 6$ & $7 \cdot 3$ & $0.029^{\star *}$ \\
\hline Other & $38 \cdot 3$ & $14 \cdot 9$ & $0 \cdot 013^{\star \star}$ & $-5 \cdot 4$ & $7 \cdot 8$ & 0.495 & $5 \cdot 8$ & $9 \cdot 2$ & 0.533 \\
\hline \multicolumn{10}{|c|}{ Income (poverty income ratio) } \\
\hline \multicolumn{10}{|c|}{ Low (0-100\%; Ref.) } \\
\hline Medium $(100-200 \%)$ & $3 \cdot 0$ & $12 \cdot 4$ & 0.808 & $-2 \cdot 4$ & $4 \cdot 7$ & 0.615 & $1 \cdot 6$ & $6 \cdot 6$ & 0.806 \\
\hline High $(>200 \%)$ & $-0 \cdot 1$ & $10 \cdot 3$ & 0.994 & -0.6 & $4 \cdot 8$ & 0.899 & $8 \cdot 1$ & $6 \cdot 0$ & $0 \cdot 182$ \\
\hline \multicolumn{10}{|l|}{ Education } \\
\hline$<$ High school (Ref.) & & & & & & & & & \\
\hline High school & $4 \cdot 6$ & $9 \cdot 1$ & $0 \cdot 617$ & $1 \cdot 8$ & $6 \cdot 8$ & 0.791 & $-2 \cdot 1$ & $4 \cdot 9$ & 0.678 \\
\hline$>$ High school & $-12 \cdot 6$ & $10 \cdot 4$ & 0.230 & -0.4 & $7 \cdot 5$ & 0.954 & $-13 \cdot 3$ & $5 \cdot 6$ & $0 \cdot 220^{* *}$ \\
\hline Model intercept & $279 \cdot 5$ & $15 \cdot 9$ & $<0.001^{\star \star}$ & $175 \cdot 5$ & $8 \cdot 4$ & $<0.001^{\star *}$ & $268 \cdot 2$ & $8 \cdot 8$ & $<0.001^{\star *}$ \\
\hline \multicolumn{10}{|l|}{ Men } \\
\hline \multicolumn{10}{|l|}{ Age group (years) } \\
\hline $18-29$ (Ref.) & & & & & & & & & \\
\hline $30-49$ & $-22 \cdot 4$ & $12 \cdot 2$ & 0.072 & $9 \cdot 7$ & $6 \cdot 1$ & $0 \cdot 120$ & $5 \cdot 2$ & $8 \cdot 7$ & 0.553 \\
\hline $50-69$ & $-42 \cdot 0$ & $16 \cdot 2$ & $0 \cdot 012^{\star \star}$ & $7 \cdot 2$ & $7 \cdot 8$ & 0.359 & $-33 \cdot 7$ & $7 \cdot 9$ & $<0.001^{\star *}$ \\
\hline$>70$ & $-100 \cdot 1$ & $18 \cdot 6$ & $<0.001^{\star *}$ & $20 \cdot 1$ & $8 \cdot 8$ & $0.028^{\star *}$ & $-87 \cdot 8$ & $8 \cdot 6$ & $<0.001^{\star *}$ \\
\hline \multicolumn{10}{|l|}{ Ethnicity } \\
\hline NH white (Ref.) & & & & & & & & & \\
\hline $\mathrm{NH}$ black & $33 \cdot 8$ & $13 \cdot 0$ & $0 \cdot 012^{\star \star}$ & $-6 \cdot 0$ & $8 \cdot 1$ & 0.465 & $17 \cdot 1$ & $9 \cdot 8$ & 0.089 \\
\hline MA & $-13 \cdot 2$ & $16 \cdot 7$ & 0.432 & $-5 \cdot 9$ & $10 \cdot 8$ & 0.586 & 1.6 & $11 \cdot 2$ & 0.890 \\
\hline Other & $39 \cdot 3$ & $22 \cdot 7$ & 0.089 & $5 \cdot 9$ & $10 \cdot 0$ & 0.557 & $3 \cdot 2$ & $14 \cdot 4$ & 0.822 \\
\hline Income (poverty income & & & & & & & & & \\
\hline Low $(0-100 \%$; Ref.) & & & & & & & & & \\
\hline Medium (100-200\%) & $-9 \cdot 3$ & $24 \cdot 2$ & 0.702 & $-13 \cdot 7$ & $8 \cdot 4$ & 0.109 & $10 \cdot 5$ & $14 \cdot 6$ & 0.476 \\
\hline High $(>200 \%)$ & $-13 \cdot 0$ & $20 \cdot 1$ & 0.522 & $-11 \cdot 4$ & $7 \cdot 5$ & 0.135 & $16 \cdot 2$ & $13 \cdot 2$ & 0.226 \\
\hline Education & & & & & & & & & \\
\hline$<$ High school (Ref.) & & & & & & & & & \\
\hline High school & $0 \cdot 7$ & $16 \cdot 4$ & 0.965 & $-1 \cdot 0$ & $9 \cdot 7$ & 0.916 & $-10 \cdot 4$ & $8 \cdot 6$ & 0.234 \\
\hline$>$ High school & $-15 \cdot 1$ & $17 \cdot 1$ & 0.381 & $-8 \cdot 4$ & $10 \cdot 6$ & 0.429 & $-26 \cdot 6$ & $9 \cdot 7$ & $0.009^{* *}$ \\
\hline Model intercept & $312 \cdot 8$ & $29 \cdot 3$ & $<0.001^{\star \star}$ & $193 \cdot 3$ & $11 \cdot 2$ & $<0 \cdot 001^{\star \star}$ & $276 \cdot 5$ & $14 \cdot 0$ & $<0.001^{\star *}$ \\
\hline Women & & & & & & & & & \\
\hline Age group (years) & & & & & & & & & \\
\hline 18-29 (Ref.) & & & & & & & & & \\
\hline $30-49$ & $20 \cdot 3$ & $7 \cdot 4$ & $0.009^{\star *}$ & $14 \cdot 4$ & $8 \cdot 1$ & 0.084 & $-3 \cdot 0$ & $6 \cdot 8$ & 0.661 \\
\hline $50-69$ & $3 \cdot 2$ & $7 \cdot 3$ & 0.668 & $16 \cdot 7$ & $9 \cdot 0$ & 0.071 & $-15 \cdot 3$ & $7 \cdot 7$ & 0.052 \\
\hline$>70$ & $-21 \cdot 2$ & $8 \cdot 5$ & $0 \cdot 016^{\star *}$ & $13 \cdot 6$ & $9 \cdot 5$ & $0 \cdot 161$ & $-36 \cdot 8$ & $7 \cdot 4$ & $<0.001^{\star *}$ \\
\hline Ethnicity & & & & & & & & & \\
\hline NH white (Ref.) & & & & & & & & & \\
\hline $\mathrm{NH}$ black & $34 \cdot 2$ & $6 \cdot 5$ & $<0.001^{\star \star}$ & $20 \cdot 0$ & $10 \cdot 9$ & 0.073 & $46 \cdot 3$ & $5 \cdot 8$ & $<0.001^{\star \star}$ \\
\hline MA & $7 \cdot 6$ & $6 \cdot 4$ & 0.241 & $21 \cdot 1$ & $20 \cdot 2$ & 0.303 & $31 \cdot 4$ & $7 \cdot 0$ & $<0.001^{\star \star}$ \\
\hline Other & $36 \cdot 7$ & $17 \cdot 2$ & $0.038^{\star *}$ & $-17 \cdot 3$ & $9 \cdot 2$ & 0.067 & $8 \cdot 1$ & $9 \cdot 3$ & 0.389 \\
\hline Income (poverty income & & & & & & & & & \\
\hline Low $(0-100 \%$; Ref.) & & & & & & & & & \\
\hline Medium (100-200\%) & $9 \cdot 9$ & $10 \cdot 2$ & 0.335 & $4 \cdot 5$ & $6 \cdot 8$ & 0.514 & $-6 \cdot 1$ & $7 \cdot 5$ & 0.417 \\
\hline High $(>200 \%)$ & $8 \cdot 8$ & $10 \cdot 2$ & 0.393 & $5 \cdot 8$ & $7 \cdot 7$ & 0.452 & $2 \cdot 0$ & $5 \cdot 7$ & 0.724 \\
\hline Education & & & & & & & & & \\
\hline$<$ High school (Ref.) & & & & & & & & & \\
\hline High school & $6 \cdot 8$ & $10 \cdot 1$ & 0.502 & $4 \cdot 7$ & $8 \cdot 7$ & 0.591 & $4 \cdot 2$ & $6 \cdot 4$ & 0.510 \\
\hline$>$ High school & $-10 \cdot 6$ & $12 \cdot 3$ & 0.393 & $7 \cdot 3$ & $11 \cdot 0$ & 0.513 & 0.0 & $6 \cdot 4$ & 0.994 \\
\hline Model intercept & $151 \cdot 7$ & $10 \cdot 3$ & $<0.001^{\star *}$ & $153 \cdot 0$ & $11 \cdot 6$ & $<0.001^{\star *}$ & $169 \cdot 0$ & $9 \cdot 4$ & $<0.001^{* *}$ \\
\hline
\end{tabular}

SES, socio-economic status; NHANES, National Health and Nutrition Examination Survey; CSFII, Continuing Survey of Food Intakes by Individuals; Ref., reference group; $\mathrm{NH}$, non-Hispanic; MA, Mexican American.

tThe differences were tested using linear regression models. Separate models were fit for each gender-survey group in each survey. The value of $\beta$ shows the difference compared to the reference group and adjusted for other variables in the model.

¥Sample sizes for NHANES III were smaller than Table 1 because of missing data on sociodemographic variables (men and women $n$ 15 105; men $n 7103$; women $n$ 8002), but were similar to Table 1 for CSFII and NHANES 1999-2004.

${ }^{* *} P<0.05$. 
Table 4 Association between meat consumption $(\mathrm{g} / \mathrm{d})$ and food purchasing factors, dietary knowledge, food label-related practics and food habit scales (age range: 20-65 years); CSFII/DHKS 1994-1996

\begin{tabular}{|c|c|c|c|c|c|c|c|c|c|c|c|}
\hline \multirow[b]{2}{*}{ Outcome variable } & \multirow[b]{2}{*}{ Independent variables } & \multicolumn{2}{|c|}{ Total meat } & \multicolumn{2}{|c|}{ Red meat } & \multicolumn{2}{|c|}{ Poultry } & \multicolumn{2}{|c|}{ Seafood } & \multicolumn{2}{|c|}{ Other meat } \\
\hline & & $\beta$ & SE & $\beta$ & SE & $\beta$ & SE & $\beta$ & SE & $\beta$ & SE \\
\hline \multicolumn{12}{|c|}{ Model At $(n$ 4263) } \\
\hline \multicolumn{12}{|c|}{ Food purchasing factors: concern about } \\
\hline Food safety & & $6 \cdot 1$ & $8 \cdot 1$ & -0.6 & $2 \cdot 4$ & $-0 \cdot 4$ & $2 \cdot 8$ & $2 \cdot 1$ & $1 \cdot 7$ & 4.5 & $7 \cdot 0$ \\
\hline Nutritional value & & $-23 \cdot 1$ & $8 \cdot 7^{\star \star}$ & $-4 \cdot 2$ & $2 \cdot 3$ & $-2 \cdot 5$ & $2 \cdot 5$ & $-1 \cdot 1$ & 1.5 & $-13 \cdot 0$ & $7 \cdot 1$ \\
\hline Food price & & $6 \cdot 0$ & $5 \cdot 7$ & $2 \cdot 5$ & $1 \cdot 6$ & $0 \cdot 4$ & $1 \cdot 4$ & $1 \cdot 0$ & 0.8 & 1.9 & $5 \cdot 9$ \\
\hline How well food keeps & & $-3 \cdot 4$ & $6 \cdot 0$ & $2 \cdot 7$ & $1 \cdot 7$ & $1 \cdot 2$ & 1.5 & -0.6 & $1 \cdot 4$ & $-6 \cdot 9$ & $5 \cdot 5$ \\
\hline Ease of preparation & & $-4 \cdot 8$ & $5 \cdot 3$ & $-2 \cdot 4$ & $1 \cdot 6$ & $0 \cdot 1$ & $1 \cdot 1$ & 0.0 & $1 \cdot 0$ & $-2 \cdot 6$ & $5 \cdot 0$ \\
\hline Food taste & & $19 \cdot 7$ & $10 \cdot 5$ & $5 \cdot 3$ & $2 \cdot 2^{\star \star}$ & $2 \cdot 3$ & $3 \cdot 1$ & $-1 \cdot 8$ & $2 \cdot 3$ & $12 \cdot 5$ & $9 \cdot 1$ \\
\hline \multicolumn{12}{|l|}{ Model $\mathrm{B} \ddagger(n$ 3973) } \\
\hline \multicolumn{12}{|l|}{ Dietary knowledge } \\
\hline $\begin{array}{l}\text { Scale } 1 \\
\text { Model C } \neq(n 2899)\end{array}$ & Perceived benefit of diet quality & $-4 \cdot 2$ & $2 \cdot 3$ & $-1 \cdot 5$ & $0.7^{\star *}$ & $0 \cdot 4$ & 0.6 & 0.9 & 0.5 & $-3 \cdot 6$ & $2 \cdot 1$ \\
\hline \multicolumn{12}{|c|}{ Dietary knowledge and label-related practices } \\
\hline Scale 1 & Perceived benefit of diet quality & $-2 \cdot 8$ & $4 \cdot 2$ & -0.9 & $1 \cdot 2$ & -0.1 & 0.9 & 0.4 & 0.8 & $-1 \cdot 8$ & 3.6 \\
\hline Scale 2 & Use of food labels and their parts & $-7 \cdot 2$ & $5 \cdot 7$ & $-4 \cdot 0$ & $1 \cdot 6^{\star \star}$ & $-3 \cdot 2$ & $1 \cdot 5^{\star *}$ & $0 \cdot 1$ & 0.8 & $0 \cdot 1$ & $5 \cdot 2$ \\
\hline Scale 3 & Look at labels: nutrients & -0.9 & $5 \cdot 8$ & 0.4 & $1 \cdot 2$ & 1.5 & $1 \cdot 0$ & 0.9 & $1 \cdot 4$ & $-3 \cdot 5$ & $5 \cdot 0$ \\
\hline Scale 4 & Look at labels: food types & $-4 \cdot 2$ & $3 \cdot 5$ & -0.9 & 0.9 & $0 \cdot 2$ & 0.7 & $-1 \cdot 3$ & 0.8 & $-2 \cdot 2$ & $3 \cdot 4$ \\
\hline Scale 5 & Understand labels and their parts/messages & $3 \cdot 1$ & $2 \cdot 8$ & $1 \cdot 7$ & $0 \cdot 8^{\star \star}$ & -0.4 & 0.8 & 0.8 & 0.6 & 1.9 & $2 \cdot 0$ \\
\hline Scale 6 & Confident in using labels & $-1 \cdot 0$ & $2 \cdot 9$ & $-1 \cdot 1$ & 0.6 & $0 \cdot 1$ & 0.7 & 0.9 & 0.8 & $-1 \cdot 2$ & $2 \cdot 5$ \\
\hline \multirow{2}{*}{\multicolumn{12}{|c|}{$\begin{array}{l}\text { Model } \mathbf{D} \neq(n 3779) \\
\text { Dietary knowledge and food habits }\end{array}$}} \\
\hline & & & & & & & & & & & \\
\hline Scale 1 & Perceived benefit of diet quality & $-0 \cdot 4$ & $2 \cdot 9$ & -0.2 & 0.9 & $0 \cdot 2$ & $0 \cdot 7$ & $1 \cdot 2$ & $0 \cdot 5^{\star \star}$ & $-1 \cdot 5$ & $2 \cdot 7$ \\
\hline Scale 7 & Food habits (e.g. low fat) & $-1 \cdot 0$ & $4 \cdot 1$ & $-2 \cdot 0$ & $1 \cdot 1$ & $2 \cdot 9$ & $0 \cdot 8^{\star *}$ & -0.8 & 0.9 & -0.2 & 3.7 \\
\hline Scale 8 & Meat group-related healthy habits & $-23 \cdot 4$ & $3 \cdot 6^{\star \star}$ & $-5 \cdot 9$ & $1 \cdot 2^{\star *}$ & $-3 \cdot 2$ & $1 \cdot 3^{\star *}$ & -0.8 & 0.6 & $-13 \cdot 0$ & $3 \cdot 2^{\star \star}$ \\
\hline
\end{tabular}

Associations are based on linear regression models. Scales are principal components analysis scores of several questions (5-11 items) and each item was measured on a 4-point Likert scale: e.g. $4=$ strongly agree $v$. $1=$ strongly disagree.

Food purchasing factors are single questions measured on a 4-point Likert scale.

tEach model controlled for age, sex, ethnicity, education and poverty income ratio.

$\ddagger$ Each model controlled for age, sex, ethnicity, education, poverty income ratio and food purchasing factors.

${ }^{\star *} P<0.05$

1988-1994 and 1999-2004. The changes between 19881994 and 1999-2004 based on NHANES data were not significant. Between 1994-1996 (CSFII) and 1999-2004 (NHANES), average MC increased in men, but decreased in women, mainly due to the decreased consumption of red meat and other meat products. This gender difference may be explained by gender differences in health and nutrition awareness and belief ${ }^{(17)}$. We suspect that some of the differences revealed by these CSFII and NHANES data are due to the true changes over time, while some may be due to differences in the samples, dietary assessment approaches and data coding across the these surveys.

Some of our observed trends in MC may reflect changes in Americans' eating behaviours over the past two decades. For example, in the 1980s and 1990s, lower MC and higher grain consumption were considered part of a healthy diet helping to reduce risks for $\mathrm{CVD}^{(1,7,11,12)}$, while during recent years reduced carbohydrate intake and higher MC have been advocated for weight loss and for reducing obesity and type 2 diabetes risks ${ }^{(2,3)}$. Increased MC over time is a worldwide phenomenon, especially during recent years, among those developing countries that have enjoyed rapid economic development. In China, e.g., national nutrition surveys show that among reference men, MC increased from $58 \mathrm{~g}$ in 1992 to
$79 \mathrm{~g}$ in $2002^{(20)}$. However, recent findings in the global climate change area implicate high-meat diets as less sustainable ${ }^{(21,22)}$.

We observed some noticeable differences in the recent changes (1994-1996 to 1999-2004) across the US ethnic and SES groups. For example, NH-black men had the largest increase in the consumption of total meat, poultry and seafood, while MA men had the smallest increase in poultry and seafood intake and their increase in red MC was comparable to the other two main ethnic groups. The ethnic differences in meat intake trends were more remarkable in women. During this period, $\mathrm{NH}$-white women reduced their consumption of all meat and 'other meat products', whereas NH-black women increased their consumption of all meat, poultry and seafood while reducing their consumption of 'other meat product'. MA women had a major reduction in 'other meat product' consumption.

In general, SES differences in consumption levels and time shifts were small and inconsistent. This is in contrast with results from other studies in other countries that found positive associations with income and education ${ }^{(17)}$, including an inverse association between income and $\mathrm{MC}^{(23,24)}$. The Glasgow and MONICA surveys revealed that VF and oily fish consumption increased significantly during 1986-1995, but showed no change 
Table 5 Associationt between meat consumption $(100 \mathrm{~g} / \mathrm{d})$ and total energy intake $(\mathrm{kcal} / \mathrm{d}) \ddagger$

\begin{tabular}{|c|c|c|c|c|c|c|c|c|c|c|c|c|c|c|c|}
\hline & \multicolumn{5}{|c|}{ NHANES $1988-1994$ ( $n$ 15 105) } & \multicolumn{5}{|c|}{ CSFII 1994-1996 (n 9596) } & \multicolumn{5}{|c|}{ NHANES 1999-2004 ( $n$ 14690) } \\
\hline & $\beta$ & SE & $P$ value & $R^{2}$ & Partial $R^{2}$ & $\beta$ & SE & $P$ value & $R^{2}$ & Partial $R^{2}$ & $\beta$ & SE & $P$ value & $R^{2}$ & Partial $R^{2}$ \\
\hline $\begin{array}{l}\text { Model } 1 \S \\
\text { All meat }\end{array}$ & $156 \cdot 1$ & $9 \cdot 1$ & $<0.001$ & 0.3367 & $0 \cdot 1015$ & 264.1 & 8.4 & $<0.001$ & $0 \cdot 2590$ & 0.2588 & 149.2 & 6.8 & $<0.001$ & 0.2958 & 0.0869 \\
\hline Model $2 \S$ & r & ( & & 0.3695 & $0 \cdot 1343$ & 204.1 & 0.4 & 0.001 & $0 \cdot 2941$ & 0.2939 & 145.2 & 0.0 & 0.001 & 0.3221 & $0 \cdot 1132$ \\
\hline Seafood & 236.0 & $14 \cdot 0$ & $<0.001$ & & & $269 \cdot 7$ & 26.5 & $<0.001$ & & & $199 \cdot 8$ & 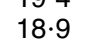 & $<0.001$ & & \\
\hline Other meat products & $119 \cdot 4$ & $8 \cdot 1$ & $<0.001$ & & & $227 \cdot 1$ & 9.8 & $<0.001$ & & & $143 \cdot 6$ & 6.9 & $<0.001$ & & \\
\hline Model $3 \S$ & & & & 0.5921 & 0.3569 & & & & 0.5591 & 0.5589 & & & & 0.5411 & 0.3322 \\
\hline Other meat products & 143.0 & $7 \cdot 3$ & $<0.001$ & & & $205 \cdot 6$ & 8.6 & $<0.001$ & & & $166 \cdot 7$ & $7 \cdot 3$ & $<0.001$ & & \\
\hline Fruits and vegetables & $64 \cdot 8$ & 3.8 & $<0.001$ & & & $46 \cdot 1$ & $3 \cdot 1$ & $<0.001$ & & & $52 \cdot 0$ & $2 \cdot 8$ & $<0.001$ & & \\
\hline Grains & $167 \cdot 7$ & $5 \cdot 7$ & $<0.001$ & & & $195 \cdot 2$ & $14 \cdot 3$ & $<0.001$ & & & $177 \cdot 1$ & $4 \cdot 4$ & $<0.001$ & & \\
\hline Model $4 \S$ & & & & & & & & & & & & & & & \\
\hline $\begin{array}{l}\text { Fruits and vegetables } \\
\text { Grains }\end{array}$ & $\begin{array}{r}71 \cdot 0 \\
153 \cdot 7\end{array}$ & $\begin{array}{l}4 \cdot 6 \\
6 \cdot 2\end{array}$ & $\begin{array}{l}<0.001 \\
<0.001\end{array}$ & 0.4407 & $0 \cdot 2055$ & $\begin{array}{r}55 \cdot 7 \\
211 \cdot 7\end{array}$ & $\begin{array}{l}3.4 \\
7.5\end{array}$ & $\begin{array}{l}<0.001 \\
<0.001\end{array}$ & 0.3244 & 0.3242 & $\begin{array}{r}54 \cdot 7 \\
165 \cdot 4\end{array}$ & $\begin{array}{l}2 \cdot 9 \\
5 \cdot 0\end{array}$ & $<0.001$ & 0.4073 & $0 \cdot 1984$ \\
\hline Model $5 \S$ & & & & & & & & & & & 165 & & & & \\
\hline $\begin{array}{l}\text { Fruits and vegetables } \\
\text { Model } 6 \$\end{array}$ & $70 \cdot 9$ & $5 \cdot 4$ & $<0.001$ & $0 \cdot 2912$ & 0.0560 & $69 \cdot 5$ & $4 \cdot 7$ & $<0.001$ & 0.0589 & 0.0587 & $60 \cdot 3$ & $3 \cdot 4$ & $<0.004$ & 0.2582 & 0.0493 \\
\hline
\end{tabular}

NHANES, National Health and Nutrition Examination Survey: CSFII, Continuing Survey of Food Intakes by Individuals.

tEach model controlled for age, sex, ethnicity, education and poverty income ratio.

$¥ 1 \mathrm{kcal}=4 \cdot 184 \mathrm{~kJ}$.

$\S$ The value of the $R^{2}$ shows the proportion of the variance in total energy intake that could be explained by all the variables included in the model. The value of the partial $R^{2}$ shows the proportion of the variance in total energy intake that could be explained by the meat and/or fruits and vegetables or grains variables included in the model. 
among the relatively deprived groups ${ }^{(25)}$. We observed increases in total and red MC among US men and more so in the higher-income group, as well as a small decrease in red MC among high-income women. This may be due to the difference in the prices of meat and VF, people's perception of their health values and response to nutrition education.

We also compared the associations between TEI and MC to those with other food groups as well as the overtime changes in the associations. Our findings suggest that interventions targeting $\mathrm{MC}$ rather than VF might have a greater impact on TEI, since MC contributed more energy and explained a higher proportion of the variation in TEI than VF, although it is important to promote VF consumption for other health benefits. The proportion of variance in TEI that could be explained by MC and VF dropped over time, suggesting the increasingly important role in the American diet played by other food groups such as snacks and sweetened beverages. For example, the CSFII data showed large increases in Americans' energy intake from salty snacks, soft drinks and pizza between 1977 and $1996^{(26)}$. Americans' average energy intake from sweetened beverages increased by $135 \%$ between 1977 and $2001^{(27)}$.

Moreover, our comparisons may indicate concerns regarding the comparability of the CSFII and NHANES dietary data, although other researchers have reported time trends in Americans' dietary intakes using these data ${ }^{(27)}$. Our findings show that one may reach different conclusions regarding the time trends based on different data sets. For example, the amount of energy contributed per $100 \mathrm{~g}$ of red meat was relatively stable between NHANES III and 1999-2004 (1443 v. 1410 kJ (345 v. $337 \mathrm{kcal}$, respectively), in model 3), much lower than what is shown in CSFII ( $2088 \mathrm{~kJ}$ ( $499 \mathrm{kcal})$ ). Thus, if one only compares the CSFII 1994-1996 and NHANES 1999-2004 data, one might conclude that red meat and grain consumption would have contributed less energy over time (e.g. a trend that can be explained by reduced fat and sugar contents). This trend, however, was not supported by the comparison between NHANES III 1988-1994 and 1999-2004 data. We suspect that part of these differences could be due to the between-survey differences in the food composition table used, sampling designs, degree of under-reporting and food grouping system, particularly with regard to mixed dishes.

We suspect that the reduction in MC, in particular among US men, may be related to the BSE (bovine spongiform encephalopathy or mad cow disease) epidemic in the United Kingdom, which peaked in January 1993 at almost 1000 new cases per week in cattle ${ }^{(28)}$. BSE created great anxiety in the United Kingdom and many other parts of the world including the United States in the mid-1990s (e.g. see Strom ${ }^{(29)}$ ). The study conducted by Burton and Young ${ }^{(30,31)}$ indicates that the first wave of media reports about BSE (from 1989 to 1995) had an immediate and detrimental impact on people's beef consumption in the United Kingdom. However, US women's red MC did not change much across the three survey periods, while the big reduction in the mid-1990s and a later increase in red MC in the early 2000s were only observed in US men. On the one hand, one may suspect that both US men and women's beef consumption would decrease beyond the mid-1990s if people were concerned about BSE. On the other hand, it is possible that because, in general, men were more likely than women to consume steaks, hamburgers and other beef products before the outbreak of the disease in Europe; they had a greater potential (both the possibility and amount) to reduce their consumption beyond that point in time. Another possible reason is that unlike Europe, in the United States, animal brains are not used to produce animal feeds. Thus, the risk of contamination is much less, and this was emphasised by the Food and Drug Administration during the scare. Further research is needed to help fully understand the reasons for the changes in people's MC that we observed.

Although it is widely believed that individuals' eating patterns are affected by many psychosocial-behavioral factors, we found that only a few of the factors we examined were associated with the total and subtypes of meat intake. This is likely due to the possible considerable day-to-day variations in individuals' MC, and the $24 \mathrm{~h}$ recall dietary data we used cannot adequately measure their usual food patterns. Furthermore, people's food patterns may be affected by many other factors including contextual-environmental factors such as food availability and food price. Nevertheless, our analysis shows that factors such as concern about food taste were associated with increased red meat intake, while concern about nutrition, perceived benefit of diet quality and use of food labels were associated with reduced red MC. Healthy habits such as trimming fat from red meat and removing skin from poultry were associated with reduced total MC.

Earlier research including ours using the DHKS suggested that nutrition knowledge was positively associated with a number of diet quality indicators and indices independently of socio-economic and demographic factors $^{(17)}$, while certain healthy eating behaviours (including trimming fat from meat and removing skin from chicken) were associated with reduced fat and saturated fat intake ${ }^{(32)}$. Food label use seemed to modify the positive association between household income and dietary quality, whereby participants who did not use food labels did not show the benefit ${ }^{(33)}$. Altogether, these findings indicate the importance of nutrition education and empowering individuals to use food labels to promote healthy eating.

While our present analysis revealed some encouraging signs of small desirable changes in Americans' MC, particularly among women with regard to red meat and 
seafood consumption, one of our earlier studies shows that Americans' VF consumption has not increased based on the NHANES III (1988-1994) and 1999-2002 data, despite the many national efforts to encourage VF consumption ${ }^{(34)}$. We found that roughly $89 \%$ of Americans failed to meet the USDA Dietary Guidelines for VF, but that the high-income group was $65 \%$ more likely to meet the guidelines than the low-income group. These suggest that more vigorous intervention efforts and policy changes are needed to promote healthy eating among Americans. In addition, the differences we observed in the relationships between SES and the consumption of meat and VF indicate potential future directions for interventions including government policies on food subsidies and taxes.

An important strength of the present study is the use of nationally representative data and tests of differences across population groups. However, it has several limitations. First, only one or two $24 \mathrm{~h}$ dietary recalls were collected in the CSFII and NHANES. Multiple $24 \mathrm{~h}$ recalls are needed to measure individuals' usual dietary intake, although there is also some evidence that under certain circumstances one recall can provide a good estimate ${ }^{(35,36)}$. Our recent sensitivity analysis assessing differences in estimates of intakes using one compared with two recalls for NHANES 1999-2004 showed a high correlation $(>0.95)$ between the two methods for dairy-related nutrients and $\mathrm{TEI}^{(37)}$. Some earlier studies have suggested an under-reporting problem in national dietary surveys using $24 \mathrm{~h}$ recalls such as NHANES; for example, mean energy intake was found to be lower than energy requirements in $15 \%$ of all 24-h recalls ${ }^{(38-40)}$. Such measurement errors might have affected our findings, including lowering the average amount of MC. We cannot rule out the possibility that some of the time trends we observed between 1994-1996 and 1999-2004 may be due to the sample and methodological differences between CSFII and NHANES. Further research is needed to answer such questions.

In conclusion, the present study provides some evidence of the shifts in food consumption patterns in the United States over the two decades, and the variation across ethnic and SES groups. The differences in the relationships between SES and the consumption of meat and VF may indicate potential future interventions. Vigorous intervention efforts and policy changes are needed to promote healthy eating among Americans.

\section{Acknowledgements}

The study was supported in part by the Johns Hopkins Center for a Livable Future, the US Department of Agriculture (2044-05322), the NIDDK/NIH (R01DK81335-01A1), the NICHD/NIH (R03HD058077-01A1, R03HD056073) and the Intramural Research Program of the National Institute on Aging (NIA/NIH/IRP). Y.W. contributed to the conceptualisation, statistical analysis, literature review, interpretation of results and write-up of manuscript. M.A.B. helped the data management and statistical analysis, interpretation of results, literature review and writeup of the manuscript. B.C., T.L.G and R.L. interpreted the results and revised the manuscript. The authors declare that they have no conflict of interest.

\section{References}

1. US Department of Health and Human Services and US Department of Agriculture (2005) Dietary Guidelines for Americans. http://www.health.gov/DietaryGuidelines/dga 2005/document/default.htm (accessed July 2008).

2. Atkins RC (editor) (2004) Atkins for Life: The Complete Controlled Carb Program for Permanent Weight Loss and Good Health. New York: St Martins Press.

3. Gardner CD, Kiazand A, Alhassan S et al. (2007) Comparison of the Atkins, Zone, Ornish, and LEARN diets for change in weight and related risk factors among overweight premenopausal women: the A to Z Weight Loss Study: a randomized trial. JAMA 297, 969-977.

4. Foster GD, Wyatt HR, Hill JO et al. (2003) A randomized trial of a low-carbohydrate diet for obesity. $N$ Engl J Med 348, 2082-2090.

5. Song Y, Manson JE, Buring JE et al. (2004) A prospective study of red meat consumption and type 2 diabetes in middle-aged and elderly women: the Women's Health Study. Diabetes Care 27, 2108-2115.

6. Leitzmann C (2005) Vegetarian diets: what are the advantages? Forum Nutr 147-156.

7. Sabate J (2003) The contribution of vegetarian diets to human health. Forum Nutr 56, 218-220.

8. Malik VS \& Hu FB (2007) Popular weight-loss diets: from evidence to practice. Nat Clin Pract Cardiovasc Med 4, 34-41.

9. McClernon FJ, Yancy WS Jr, Eberstein JA et al. (2007) The effects of a low-carbohydrate ketogenic diet and a low-fat diet on mood, hunger, and other self-reported symptoms. Obesity (Silver Spring) 15, 182-187.

10. Togo P, Osler M, Sorensen TI et al. (2001) Food intake patterns and body mass index in observational studies. Int $J$ Obes Relat Metab Disord 25, 1741-1751.

11. Astrup A, Meinert Larsen T \& Harper A (2004) Atkins and other low-carbohydrate diets: hoax or an effective tool for weight loss? Lancet 364, 897-899.

12. Dansinger ML, Gleason JA, Griffith JL et al. (2005) Comparison of the Atkins, Ornish, Weight Watchers, and Zone diets for weight loss and heart disease risk reduction: a randomized trial. JAMA 293, 43-53.

13. Centers for Disease Control and Prevention (2006) NHANES: data sets and related documentation. http://www. cdc.gov/nchs/about/major/nhanes/datalink.htm (accessed May 2006).

14. Centers for Disease Control and Prevention (1996) The Third National Health and Nutrition Examination Survey (NHANES III 1988-1994) Reference Manuals and Reports $(C D-R O M)$. Bethesda, MD: Centers for Disease Control and Prevention.

15. US Department of Agriculture ARS FSRG (1994-1996) Data (CD-ROM) and Documentation for the 1994-1996 Continuing Survey of Food Intakes by Individuals (CSFII)-Diet and Health Knowledge Survey. National Technical Information Service Accession no. PB98-500457. Washington, DC: USDA.

16. Forshee RA \& Storey ML (2006) Demographics, not beverage consumption, is associated with diet quality. Int J Food Sci Nutr 57, 494-511.

17. Beydoun MA \& Wang Y (2008) How do socio-economic status, perceived economic barriers and nutritional 
benefits affect quality of dietary intake among US adults? Eur J Clin Nutr 62, 303-313.

18. Williams DR \& Collins C (1995) US socioeconomic and racial differences in health: patterns and explanations. Ann Rev Sociol 21, 349-386.

19. STATA (2005) Statistics/Data Analysis: Release 9, 9.0 ed. College Station, TX: Stata Corporation.

20. Zhai FY, He YN, Ma GS et al. (2005) Study on the current status and trend of food consumption among Chinese population. Zhonghua Liu Xing Bing Xue Za Zbi 26, 485-488.

21. Steinfeld H, Gerber P, Wassenaar T et al (2006). Livestock's Long Shadow: Environmental Issues and Options. Rome: Livestock, Environment and Development \& Food and Agricultural Organization of the United Nations.

22. Horrigan L, Lawrence RS \& Walker P (2002) How sustainable agriculture can address the environmental and human health harms of industrial agriculture. Environ Rep 110, 445-456.

23. Moreira PA \& Padrao PD (2004) Educational and economic determinants of food intake in Portuguese adults: a crosssectional survey. BMC Public Health 4, 58.

24. Hulshof KF, Brussaard JH, Kruizinga AG et al. (2003) Socioeconomic status, dietary intake and 10 y trends: the Dutch National Food Consumption Survey. Eur J Clin Nutr 57, $128-137$.

25. Wrieden WL, Connaghan J, Morrison C et al. (2004) Secular and socio-economic trends in compliance with dietary targets in the north Glasgow MONICA population surveys 1986-1995: did social gradients widen? Public Health Nutr 7, 835-842.

26. Nielsen SJ, Siega-Riz AM \& Popkin BM (2002) Trends in energy intake in US between 1977 and 1996: similar shifts seen across age groups. Obes Res 10, 370-378.

27. Nielsen SJ \& Popkin BM (2004) Changes in beverage intake between 1977 and 2001. Am J Prev Med 27, 205-210.

28. Centers for Disease Control and Prevention (2009) BSE (bovine spongiform encephalopathy or mad cow disease). http://www.cdc.gov/ncidod/dvrd/bse (accessed February 2010).

29. Strom S (1996). 'Mad Cow Disease' threatens the farming life. New York Times, 25 March.
30. Burton M \& Young $T$ (1996) The impact of BSE on the demand for beef and other meats in Great Britain. Appl Econ 28, 687-693.

31. Burton M \& Young T (1997) Measuring meat consumers' response to the perceived risks of BSE in Great Britain. Risk Decision Policy 2, 9-18.

32. Capps O Jr, Cleveland L \& Park J (2002) Dietary behaviors associated with total fat and saturated fat intake. J Am Diet Assoc 102, 490-502.

33. Perez-Escamilla R \& Haldeman L (2002) Food label use modifies association of income with dietary quality. J Nutr 132, 768-772.

34. Casagrande SS, Wang Y, Anderson C et al. (2007) Have Americans increased their fruit and vegetable intake? The trends between 1988 and 2002. Am J Prev Med 32, 257-263.

35. Basiotis PP, Welsh SO, Cronin FJ et al. (1987) Number of days of food intake records required to estimate individual and group nutrient intakes with defined confidence. $J$ Nutr 117, 1638-1641.

36. Fung TT, Hu FB, McCullough ML et al. (2006) Diet quality is associated with the risk of estrogen receptor-negative breast cancer in postmenopausal women. J Nutr 136, 466-472.

37. Beydoun MA, Gary TL, Caballero BH et al. (2008) Ethnic differences in dairy and related nutrient consumption among US adults and their association with obesity, central obesity, and the metabolic syndrome. Am J Clin Nutr 87, 1914-1925.

38. Briefel RR, McDowell MA, Alaimo K et al. (1995) Total energy intake of the US population: the third National Health and Nutrition Examination Survey, 1988-1991. Am J Clin Nutr 62, Suppl. 5, 1072S-1080S.

39. Dwyer J, Picciano MF \& Raiten DJ (2003) Estimation of usual intakes: what we eat in America-NHANES. J Nutr 133, 609S-623S.

40. Krebs-Smith SM, Graubard BI, Kahle LL et al. (2000) Low energy reporters vs others: a comparison of reported food intakes. Eur J Clin Nutr 54, 281-287. 